\title{
Edema hemorrágico agudo del lactante: reporte de un caso de presentación atípica
}

\author{
PAULA YARMUCH G. ${ }^{1}$, XIMENA CHAPARRO R. ${ }^{1}$, CECILIA FISCHER S. ${ }^{1}$, \\ CHRISTIAN HAUSSMANN G. ${ }^{2}$, SAMUEL BENVENISTE D. ${ }^{3}$ \\ 1. Médico Pediatra, Unidad de Dermatología, Hospital Dr. Exequiel González Cortés. \\ 2. Alumno $4^{\circ}$ año Medicina, Pontificia Universidad Católica de Chile. \\ 3 Médico, Servicio de Anatomía Patológica, Hospital Dr. Exequiel González Cortés.
}

\begin{abstract}
Acute hemorrhagic edema of infancy. Case report

Acute infant hemorragic edema is a benign condition that affects children between 4 month and 2 years of age. Quick onset inflammatory edema and generalized ring shaped symmetric purpura are characteristic. Histology is of leucocitoclastic vasculitis. Clinical case: 9 month old child with a respiratory tract affection 2 weeks previous, consulting for left leg edema and fever, afterwards cutaneous and enteral mucosa purpura. Discussion: Acute hemorrhagic edema is a benign and self-limited illness. The main differential diagnosis is Schönlein Henoch Purpura, and a careful evaluation and clinical follow-up is neccesary.

(Key words: Hemorrhagic edema, asymmetric edema, vasculitis).

Rev Chil Pediatr 2012; 83 (5): 462-467
\end{abstract}

\section{RESUMEN}

Introducción: El Edema Hemorrágico Agudo del Lactante (EHAL) es una condición benigna que afecta a niños entre 4 meses y 2 años, de inicio brusco, con edema inflamatorio y lesiones cutáneas del tipo purpúrico, anulares generalizadas y simétricas. A la histología los hallazgos son compatibles con una vasculitis leucocitoclástica. Caso clínico: Lactante de 9 meses de edad, que cursó con cuadro respiratorio 2 semanas previas y que consulta por aumento de volumen de la extremidad inferior izquierda y fiebre, agregándose en forma progresiva lesiones cutáneas purpúricas, compromiso de mucosas y gastrointestinal. Discusión: El EHAL es una condición benigna y autolimitada, cuyo principal diagnóstico diferencial es Púrpura de Schönlein-Henoch, la cual requiere una cuidadosa evaluación y segumiento clínico.

(Palabras clave: Edema hemorrágico, edema asimétrico, vasculitis).

Rev Chil Pediatr 2012; 83 (5): 462-467

Recibido el 24 de octubre de 2011, devuelto para corregir el 6 de junio de 2012, segunda versión el 26 de junio de 2012 , aceptado para publicación el 30 de julio de 2012.

Este trabajo cumple con los requisitos sobre consentimiento /asentimiento informado, comité de ética, financiamiento, estudios animales y sobre la ausencia de conflictos de intereses según corresponda.

Correspondencia a:

Paula Yarmuch Guzmán

E-mail: paulayarmuch@hotmail.com 


\section{Introducción}

El Edema Hemorrágico Agudo del Lactante (EHAL) es una forma infrecuente de vasculitis leucocitoclástica, presentándose como un trastorno cutáneo benigno, caracterizado clínicamente por un inicio brusco, con desarrollo de edema inflamatorio acral simétrico y la posterior aparición de lesiones cutáneas del tipo anulares purpúricas equimóticas (edematosas y dolorosas) que comprometen la piel de forma extensa y simétrica. Además, es frecuente encontrar compromiso de los pabellones auriculares, párpados, mejillas y extremidades ${ }^{1,2}$. Destaca que esta florida presentación cutánea contrasta con el buen estado general y fiebre de escasa cuantía ${ }^{3}$.

Respecto a su historia natural, es una patología autolimitada en el tiempo, con una duración promedio de 1 a 3 semanas. Afecta casi exclusivamente a niños entre los 2 meses y 2 años de edad, siendo en su mayoría lactantes del sexo masculino $(4,6: 1)^{4}$. No se ha identificado un factor racial asociado. Su etiología es desconocida, pero se ha visto una mayor incidencia en invierno ${ }^{5}$. Además se han descrito la presencia de factores gatillantes como: infecciones virales o bacterianas, inmunizaciones o ingesta previa de medicamentos ${ }^{6,7}$. Pese a que se han reportado casos en muchos países, aún no se conoce su verdadera incidencia.

El diagnóstico es fundamentalmente clínico, complementado con estudio histológico. No se han descrito tratamientos específicos para esta enfermedad. Se aconseja el manejo sintomático con uso antibióticos para el control de las infecciones subyacentes. Los corticoides sistémicos y antihistamínicos no han demostrado evidencia concluyente de haber tenido beneficio para el control del cuadro ${ }^{6-8}$.

El EHAL sigue siendo una patología que en general es poco conocida por el pediatra y además, al aparecer los signos de forma progresiva, resulta difícil plantear el diagnóstico en forma precoz y se tiende a confundir con otros patologías.

El objetivo de la presente comunicación es presentar un caso de EHAL para que el pediatra lo incluya dentro de los diagnósticos diferenciales al evaluar un paciente con púrpura.

\section{Caso clínico}

Lactante de 9 meses de edad con antecedentes de Síndrome Bronquial Obstructivo Recurrente, Ductus Arterioso Persistente y Foramen Oval, que presentó dos semanas previas al ingreso una infección respiratoria, que se manejó con salbutamol y amoxicilina. Posteriormente desarrolló edema de pie izquierdo, dolor, impotencia funcional y fiebre. Consultó al tercer día de evolución del cuadro, sospechándose una celulitis, por lo cual se inició tratamiento con cloxacilina oral. Durante las primeras $24 \mathrm{~h}$ de iniciada la terapia antimicrobiana, evolucionó con progresión del edema por lo que se decidió su hospitalización, descartándose el posible origen traumático de ésta. A las pocas horas de ingreso aparecieron pápulas y placas purpúricas rojo-violáceas edematosas en extremidades superiores y aisladas en tronco, algunas coalescentes formando lesiones anulares, evolucionando además con edema bipalpebral y placas eritemato-violáceas en pabellones auriculares (figuras 1a, 1 b y 1c). Por sospecha de reacción adversa a fármacos se cambió la terapia antimicrobiana a clindamicina y recibó una dosis de corticoides y antihistamínicos sistémicos. Se solicitó evaluación por dermatología planteándose la sospecha de EHAL. Debido al importante edema e impotencia funcional que comprometía la extremidad inferior izquierda en toda su extensión y sin lesiones cutáneas purpúricas en dicha localización, se complementó el estudio con una ecografía doppler, que descartó compromiso de grandes vasos.

Se realizó una biopsia de piel de una de las lesiones de la extremidad inferior derecha, cuyo estudio histopatológico evidenció leucocitos polimorfonucleares neutrófilos perivasculares e intersticiales en la dermis que resultaba compatible con una vasculitis leucocitoclástica (figuras 2 y 3). Dentro de su estudio de laboratorio destacaba una IgA elevada (182 $\mathrm{mg} / \mathrm{dl}$ ), siendo los otros exámenes normales: complemento, pruebas de coagulación, función renal y orina completa.

Durante su hospitalización evolucionó con lesiones purpúricas en escarapela (figura 4) y en la mucosa oral (figura 5), comprometiendo 


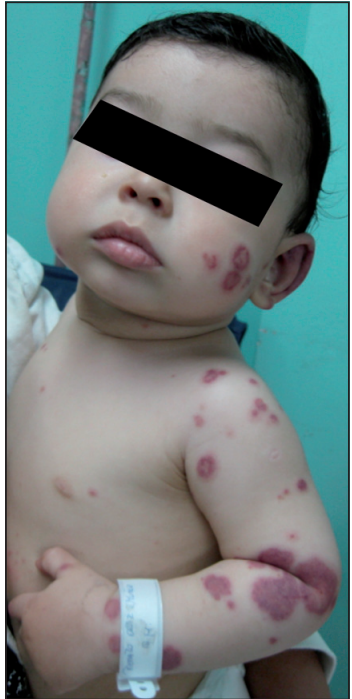

Figura 1a. Placas purpúricas edematosas anulares de predominio facial y en extremidades al ingreso hospitalario.

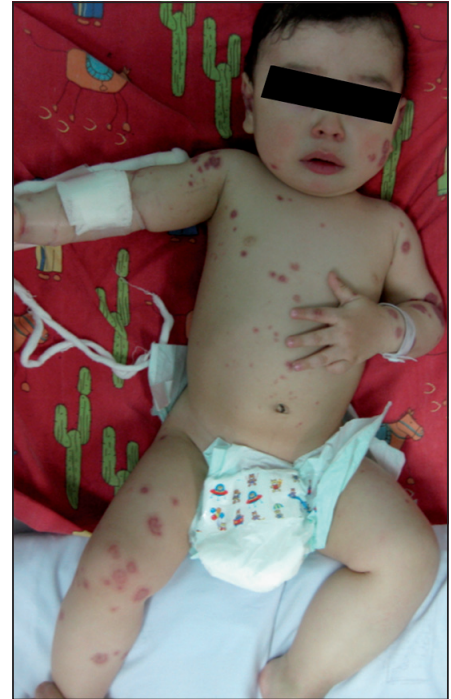

Figura 1b. Lesiones purpúricas en zona facial y extremidades, con algunas lesiones aisladas en tronco.

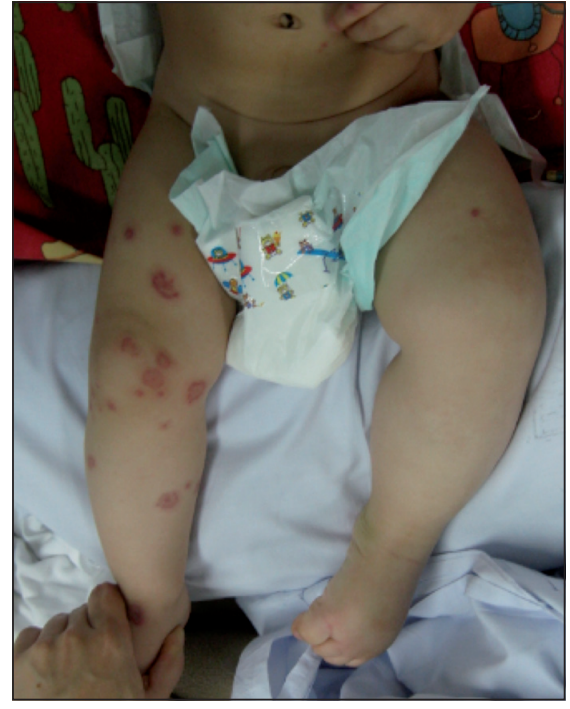

Figura 1c. Edema que compromete extremidad inferior izquierda y lesiones purpúricas extremidad inferior derecha.

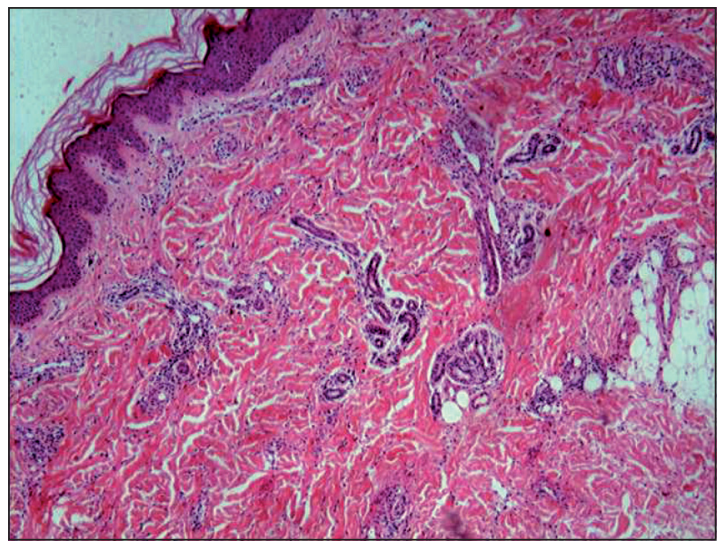

Figura 2. Histopatología. Infiltrado inflamatorio perivascular e intersticial, tinción de Hematoxilina-Eosina, aumento $10 \mathrm{X}$.

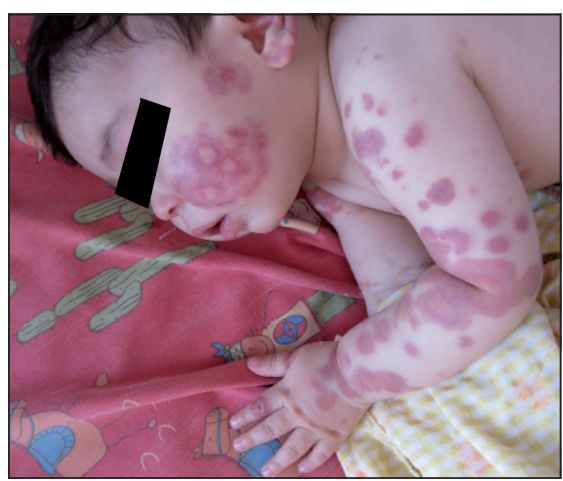

Figura 4. Lesiones purpúricas típicas y edema que compromete mejillas, pabellón auricular y extremidades superiores a las $48 \mathrm{~h}$ de hospitalización.

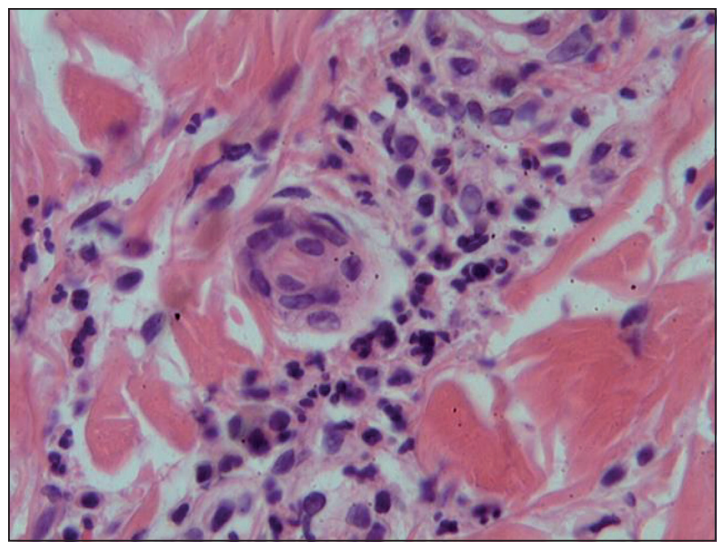

Figura 3. Histopatología. Vaso sanguíneo central rodeado de polimorfonucleares neutrófilos. Tinción de Hematoxilina-Eosina, aumento 40X.

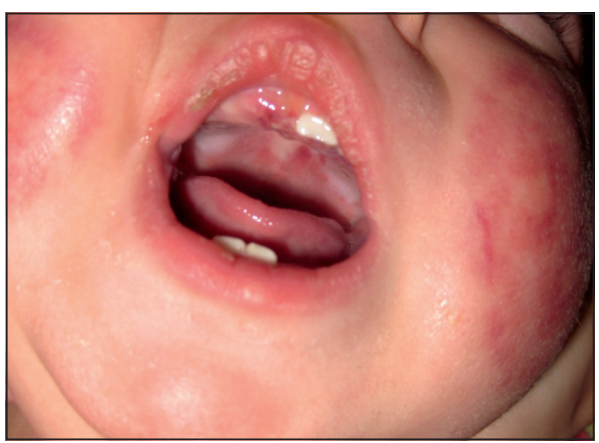

Figura 5. Lesiones purpúricas en mucosa oral. 
paladar y encía superior, además de afectar la zona perianal. Presentó además deposiciones con estrías sanguinolentas en una oportunidad, por lo que se inició metilprednisolona $20 \mathrm{mg} /$ $\mathrm{kg} /$ día vía endovenosa por 3 días con respuesta favorable y luego se continuó con prednisona $1,5 \mathrm{mg} / \mathrm{kg} /$ día vía oral por 4 días más para luego disminuirla en forma progresiva.

La paciente continúa en control en el Policlínico de Dermatología Infantil, sin recaídas en 2 años de seguimiento y con exámenes nefrológicos normales.

\section{Discusión}

E1 EHAL es un tipo de vasculitis leucocitoclástica, de carácter benigna y autolimitada. Clínicamente se caracteriza por edema simétrico con distribución acral en dorso de manos y pies que se extiende posteriormente en sentido proximal. Luego se agregan lesiones cutáneas purpúricas que comienzan como pápulas y/o placas de aspecto edematoso y violáceo, no pruriginosas, que evolucionan hacia una morfología anular o en escarapela ${ }^{1,2}$. Estas lesiones tienen un tamaño que fluctúa entre 1 y $5 \mathrm{~cm}$ de diámetro, de bordes abruptos y límites redondeados, que pueden adquirir un aspecto equimótico, comprometiendo principalmente la zona facial, pabellón auricular y extremidades. Los pacientes se encuentran en buen estado general, en ocasiones febriles y generalmente sin compromiso visceral ${ }^{9}$.

La etiología del EHAL es desconocida, aunque se piensa que podría corresponder a una enfermedad mediada por complejos inmunes. Se ha descrito la presencia de factores gatillantes hasta en el 75\% de los casos ${ }^{9}$. Dentro de éstos destacan: infecciones virales (infecciones respiratorias altas, otitis media ${ }^{10,11}$, conjuntivitis ${ }^{6,12}$ ), bacterianas (infecciones estreptocócicas ${ }^{13-15}$, tuberculosis pulmonar ${ }^{5}$, bronconeumonía ${ }^{7}$ o infecciones del tracto urinario), vacunaciones (sarampión, difteria, pertussis, tétanos o en combinación) e hipersensibilidad a medicamentos (penicilina, cefalosporinas, trimetoprim sulfametoxazol, paracetamol y otros).

El diagnóstico del EHAL es fundamental- mente clínico, siendo de gran relevancia la histología como examen complementario. En el estudio histológico aparecen cambios típicos de una vasculitis leucocitoclástica, caracterizada por infiltrado de polimorfonucleares neutrófilos con necrosis fibrinoide y extravasación de eritrocitos ${ }^{1}$. El infiltrado es de predominio perivascular y afecta exclusivamente a los vasos pequeños de la dermis ${ }^{7}$.

El diagnóstico diferencial debe establecerse con otras entidades del síndrome purpúrico en pediatría, principalmente descartando patologías graves y de peor pronóstico como la meningococcemia, eritema multiforme, dermatosis neutrofílica aguda y urticaria, entre otras.

La alta similitud en la presentación clínica del EHAL y Púrpura de Schönlein-Henoch (PSH), ha sido un punto de debate entre los especialistas, ya que no se ha podido establecer la clara diferencia entre estas 2 patologías. Es por esto que resulta importante enfocarse en el análisis diferencial entre ambas (tabla 1). Algunos autores han considerado al EHAL como una variante del PSH, con el que comparte ciertas características clínico-patológicas, como la predisposición de temporada (invierno), el pródromo, la afección cutánea y los hallazgos histopatológicos en la biopsia de piel (vasculitis leucocitoclástica). Otros consideran al EHAL como una entidad distinta, en vista de la edad de aparición más temprana, la falta de compromiso visceral (o en casos de presentarlo, éste es benigno y autolimitado), el carácter distintivo de las lesiones cutáneas, el excelente pronóstico y por último, el escaso o ausente depósito de IgA perivascular encontrado en la histología de la dermis ${ }^{1,7,16}$ (tabla 1). Se ha tratado de estudiar el solapamiento de estas dos enfermedades, con el propósito de encontrar en qué punto podrían estar relacionadas. Incluso se habla de que corresponderían a distintas formas de presentación de una misma patología ${ }^{16}$. Goraya y $\operatorname{Kaur}^{17}$, plantean que la diferencia entre el hallazgo constante de depósito de IgA en la PSH y el ocasional en el EHAL, sería debido a la inmadurez del sistema IgA del lactante, quien posee niveles séricos de IgA entre el 19-25\% del adulto, a pesar de que el depósito de IgA es inespecífico y es posible encontrarlo en otras vasculitis. 
Tabla 12,6,22

\begin{tabular}{|lll|}
\hline & EHAL & PSH \\
\hline Edad & $2-24$ meses & 3 a 7 años \\
\hline Sexo & Predominio masculino & Predominio masculino \\
\hline Estación & Invierno & Invierno \\
\hline Pródromo & Infección respiratoria, medicamentos, vacunas & Infección respiratoria, medicamentos, vacunas \\
\hline Lesiones & Petequias, equimosis, púrpuras en escarapela & Pápulo-petequial, urticarial \\
\hline Edema & Constante & Infrecuente \\
\hline Localización & Cara, pabellones auriculares y extremidades & Sobre todo miembros inferiores y glúteos. \\
\hline Afectación visceral & Excepcional y autolimitada & Ocasional afectación de escroto \\
\hline Síntomas asociados & Febrícula o fiebre de bajo grado & Frecuente \\
\hline Evolución & Benigna. Recuperación espontánea en 1 a 3 & Artralgias, dolor abdominal, hemorragia gastro- \\
\hline Recidiva & semanas & Incierta \\
\hline Vasculitis leucocitoclástica & Rara & Sí hematuria, proteinuria \\
\hline Depósito de lgA perivascular & $30 \%$ & Frecuente (50\%) \\
\hline
\end{tabular}

Por otro lado, Gatorno y cols ${ }^{18}$, publicaron un caso en que dos hermanos desarrollaron PSH y EHAL, ambos habían sido diagnosticados 20 días antes de faringitis y tratados durante 10 días con amoxicilina, con una diferencia de 4 días la niña de 3,6 años desarrolló un PSH y su hermano de 16 meses un EHAL. Toda esta evidencia apoya la idea que podría corresponder una misma enfermedad con distinta presentación según grupos etarios.

Una característica del EHAL es el infrecuente compromiso visceral ${ }^{6}$, aunque se han descrito casos con artralgias, síntomas de afectación digestiva (dolor abdominal, vómitos, diarrea serosanguinolenta, melenas, invaginación intestinal), y renal (hematuria o proteinuria aisladas y transitorias) $)^{19,20}$. La evolución de los pacientes con EHAL es favorable, con recuperación completa y espontánea en 1-3 semanas. Aunque no hay seguimientos a largo plazo, la ausencia de reportes de complicaciones sugiere su carácter benigno. Las recaídas son excepcionales, aunque han sido descri$\operatorname{tas}^{5,21}$.

El EHAL es una enfermedad benigna, autolimitada y sin complicaciones en la gran mayoría de los casos. No existe tratamiento espe- cífico para esta patología ${ }^{2,5,21}$, pero se sugiere indicar reposo y los padres deben ser educados respecto al curso benigno del cuadro ${ }^{2}$. Los antibióticos deben ser usados para las infecciones intercurrentes ${ }^{5}$. En caso de compromiso articular se recomienda el uso de antiinflamatorios ${ }^{2}$. Los corticoides sistémicos y antihistamínicos no han demostrado evidencia concluyente de ser beneficiosos para el control del EHAL ${ }^{5-9}$, a pesar de lo cual son usados frecuentemente, sobretodo cuando los síntomas gastrointestinales son persistentes o severos ${ }^{23}$, como fue el caso de nuestro paciente.

Cabe destacar que en este caso resultó difícil establecer un diagnóstico precozmente debido a la presentación clínica atípica. Nuestro paciente inició edema, dolor e impotencia funcional del pie izquierdo, progresando en forma proximal. Se hospitalizó por sospecha de patología infecciosa y posteriormente se agregaron lesiones purpúricas en cara, pabellones auriculares y extremidades. Finalmente, presentó compromiso gastrointestinal y de mucosas, siendo estos últimos poco habituales en esta entidad.

Nos parece importante que el pediatra tenga conocimiento de esta patología con el fin 
de sospechar el diagnóstico en forma precoz y no confundirla con otras enfermedades cuyo manejo podría perjudicar al lactante. Tanto los datos de la anamnesis como los hallazgos del examen físico resultan importantes en el diagnóstico de EHAL y al ser de carácter autolimitado y sin complicaciones, su manejo se basaría en el buen control de las comorbilidades. Al no existir publicaciones de seguimiento a largo plazo de pacientes con esta patología y por su similitud con el PSH, nosotros recomendamos seguimiento clínico y de laboratorio con examen de orina, microalbuminuria y función renal.

\section{Referencias}

1.- Miner I, Vivanco A, Muñoz J, Landa J, Albisu Y: Edema agudo hemorrágico del lactante. Bol. S Vasco-Nav Pediatr 2004; 37: 13-6.

2.- Pérez L, Benavides A, Barrientos B, Deza C, Guixe C, Mendoza G: Edema hemorrágico agudo del lactante. Rev Chil Pediatr.2006; 77: 599-603.

3.- Lantner RR, Ros SP: Acute hemorrhagic edema of infancy. Pediatr Emerg Care 1996; 12: 111-2.

4.- Fiore E, Rizzi M, Ragazzi M, et al: Acute hemorrhagic edema of young children (cockade purpura and edema): a case series and systematic review. J Am Acad Dermatol 2008; 59: 684-95.

5.- Gonggryp L, Todd G: Acute Hemorrhagic Edema of Childhood. Pediatric Dermatology 1998; 15: 91-6.

6.- Legrain V, Lejean S, Tai'eb A, Guillard JM, Battin J, Maleville $J$ : Infantile acute hemorrhagic edema of the skin: study of ten cases. J Am Acad Dermatol 1991; 24: 17-22.

7.- Saraclar Y, Tinaztepe K, Adalioglu G, Tuncer A: Acute hemorrhagic edema of infancy (AHEI) a variant of Henoch-Schonlein purpura or a distinct clinical entity? J Allergy Clin Immunol 1990; 86: 473-83.

8.- Ince E, Mumcu Y, Suskan E, Yakinkaya F, Tumer N, Cin $S$ : Infantile acute hemorrhagic edema: a variant of leucocytoclastic vasculitis. Pediatr Dermatol 1995; 12 : 224- 7 .
9.- $\quad$ Smitt J, Vermeer M, Faber W: Acute hemorrhagic edema of infancy (AHEI). Clinics in Dermatology 2002; 20: 2-3.

10.- Pride HB, Maroon M, Tyler WB: Ecchymoses and edema in a 4-month-old boy. Pediatr Dermatol 1995; 12: 373-5.

11.- Cox NH: Seidlmayer's syndrome: postinfectious cockade purpura of early childhood. J Am Acad Dermatol 1992; 26: 275.

12.- Krause I, Lazarov A, Rachmel A, et al: Acute haemorrhagic oedema of infancy, a benign variant of leucocytoclastic vasculitis. Acta Paediatr 1996; 85: 114-7.

13.- Sánchez del Río J, Marín MC, Martínez DN, et al: Edema agudo hemorragico del lactante. Actas Dermato-Sif 1982; 73: 129-32.

14.- Snoussi N, Strobel M, Heid E, et al: Oede'me aigu he'morragique du nourisson: de'monstration d'une vascularite allergique dermique. Arch Belg Dermatol 1973; 29: 259-60.

15.- Laugier MP: Oede'me aigu he'morragique (purpura en cocarde avec oede'me). Bull Soc Fr Dermatol Syph 1969; 76: 462-3.

16.- Shah D, Goraya J, Poddar BV: Acute infantile hemorrhagic edema and Henoch-Schönlein Purpura overlap in a child. Pediatric Dermatology 2002; 19: 92-3.

17.- Goraya J, Kaur S: Acute infantile hemorrhagic edema and Henoch-Schönlein purpura: is $\operatorname{IgA}$ the missing link?. J Am Acad Dermatol 2002; 47: 801-2.

18.- Gattorno M, Picco P, Vignola S, Di Rocco M, Buoncompagni A: Brother and sister with different vasculitides. Lancet 1999; 353: 728.

19.- Garty B, Ofer I, Finkelstein Y: Acute hemorr- hagic edema of infancy. Isr Med Assoc J 2002; 4: 228-9.

20.- Paradisi M, Annesi G, Corrado A: Infantile acute hemorrhagic edema of the skin. Cutis 2001; 68: 127-9.

21.- Millard T, Harris A, MacDonald D: Acute infantile hemorrhagic oedema. J Am Acad Dermatol 1999; 41: 837-9.

22.- Yeste D, González U, González J, De Mir I, Martín M, Castello F: Edema agudo hemorrágico del lactante. An Esp Pediatr 1993; 38: 79-81.

23.- Yu J, Mancini A, Miller M: Intussusception in an Infant with Acute Hemorrhagic Edema of Infancy Pediatric Dermatology 2007; 24 (1): 6-64. 